\title{
A short-term flare on a time scale of 20 days in the BL Lac object OT 081
}

\section{Takafumi Ishida*}

Department of Physics, Faculty of Science, Yamaguchi University, Yoshida 1677-1, Yamaguchi-city, Yamaguchi 753-8512, Japan

E-mail: takafumi.o.ishidadgmail.com

\section{Kenta Fujisawa}

Department of Physics, Faculty of Science, Yamaguchi University, Yoshida 1677-1, Yamaguchi-city, Yamaguchi 753-8512, Japan \& The Research Institute for Time Studies, Yamaguchi University, Yoshida 1677-1, Yamaguchi-city, Yamaguchi 753-8511, Japan

\section{Motoki Kino}

Institute of Space and Astronautical Science, Japan Aerospace Exploration Agency, Yoshinodai 3-1-1, Chuo-ku, Sagamihara, Kanagawa 252-5210, Japan

\section{Kotaro Niinuma}

Department of Physics, Faculty of Science, Yamaguchi University, Yoshida 1677-1, Yamaguchi-city, Yamaguchi 753-8512, Japan

\begin{abstract}
We have conducted daily monitoring of the BL Lac object OT 081 at $8.4 \mathrm{GHz}$ with the Yamaguchi $32 \mathrm{~m}$ radio telescope from February to June 2010. Based on these data, we detected a short-time flare with a duration of 19.3 days and an amplitude of $0.73 \mathrm{Jy}$, overlapped on a long-term trend with a time scale of about 200 days. This short-term flare was also observed in the University of Michigan data at 4.8, 8.0, and $14.5 \mathrm{GHz}$. Subtracting the long-term trend allowed us to study the short-term flare in details. The flare was found to occur synchronously at 8.4 and $14.5 \mathrm{GHz}$, with no significant delays between the two frequencies. This suggests that the flaring region was optically thin, as otherwise the radio emission would have peaked with a delay at the lower frequency. The duration of the flare indicates that the emitting region is smaller than $0.01 \mathrm{pc}$.
\end{abstract}

11th European VLBI Network Symposium \& Users Meeting,

October 9-12, 2012

Bordeaux, France

\footnotetext{
*Speaker.
} 


\section{Introduction}

Among various classes of active galactic nuclei (AGNs), the blazar class (consisting of BL Lacertae objects and optically violently variable quasars) is known for rapid flux variability. At optical, $\mathrm{X}$-ray, and gamma-ray bands, the time scale of flux variability is often shorter than a month because the emission emanates from small regions with a size of less than one light-month (e.g. [ए]]). In the radio band, several monitoring programs are currently running, and these provide long-term light curves of various blazars (see review in []]). The Effelsberg, IRAM, Metsähovi and Owens Valley Radio Observatory programs conduct radio flux monitoring with a sampling of 2-4 weeks. The sampling of the University of Michigan Radio Astronomy Observatory (UMRAO) program (1-2 weeks) is the shortest one. Such sampling, however, remains too sparse to study variability at intervals shorter than about 2-3 weeks. To this end, daily monitoring would be desirable.

Kadota et al. [5] conducted daily monitoring of PKS $1510-089$ at $8.4 \mathrm{GHz}$ using the Yamaguchi $32 \mathrm{~m}$ telescope and found variability with a time scale of 30 days. Since PKS 1510-089 is known to be strongly and rapidly variable in the optical, X-ray, and gamma-ray bands (e.g. [Q, प]), it is a candidate for short-term variability in the radio band. Kadota et al. [G] indeed found a clear increase of the flux density of PKS 1510-089 while in a long-term decay phase, but their monitoring ended before that short-term flare is over, preventing a detailed study of the variation pattern.

This paper reports the discovery of short-term variability in the object OT 081 (also known as PKS $1749+096$ or $4 \mathrm{C}+09.57$ ) at $\mathrm{z}=0.32$. OT 081 is one of the most extreme AGNs showing strong and rapid variability in all spectral ranges. This object is an ultra-luminous BL Lac object with an optical polarization of up to $32 \%$ [四]. Dallacasa et al. [B] classified this source as a highfrequency peaker, while Torniainen et al. [W] suggested it is a flat-spectrum source with an inverted spectrum during flares. Recent VLBI observations indicate superluminal motions with 5-21 $c$ [目]. OT 081 is thus a suitable target for intensive monitoring to explore short-term variability in blazars.

\section{Observations}

Monitoring of the flux density of OT 081 has been carried out with the Yamaguchi $32 \mathrm{~m}$ radio telescope for 148 days from 2010 February 1 (DOY 32 ${ }^{1}$ ) to 2010 June 29 (DOY 180). The observing campaign covers the same period as the campaign for 1510-089 discussed above [5]. A strong, nearby radio source $3 \mathrm{C} 348$ (6.53 Jy at $8.4 \mathrm{GHz}$, see [8]]) was selected as flux density calibrator. The calibrator and the target source were observed at the same elevation range. Hence, the flux density of OT 081 is determined relative to that of $3 \mathrm{C} 348$. The total on-source time for OT 081 was $45 \mathrm{~min} / \mathrm{day}$. The standard measurement error was typically $0.03 \mathrm{Jy}$. Due to the weather, there were only 80 effective observations. Observations were not made from 2010 May 17 (DOY 137) to 2010 June 2 (DOY 153) due to another observing program.

\section{Results and discussion}

\subsection{Detection of a short-term flare}

Figure (left) shows the light curve of OT 081 at $8.4 \mathrm{GHz}$. From the beginning of the campaign

\footnotetext{
${ }^{1}$ DOY is the day of year. DOY 0 of 2010 corresponds to MJD 55196.
} 

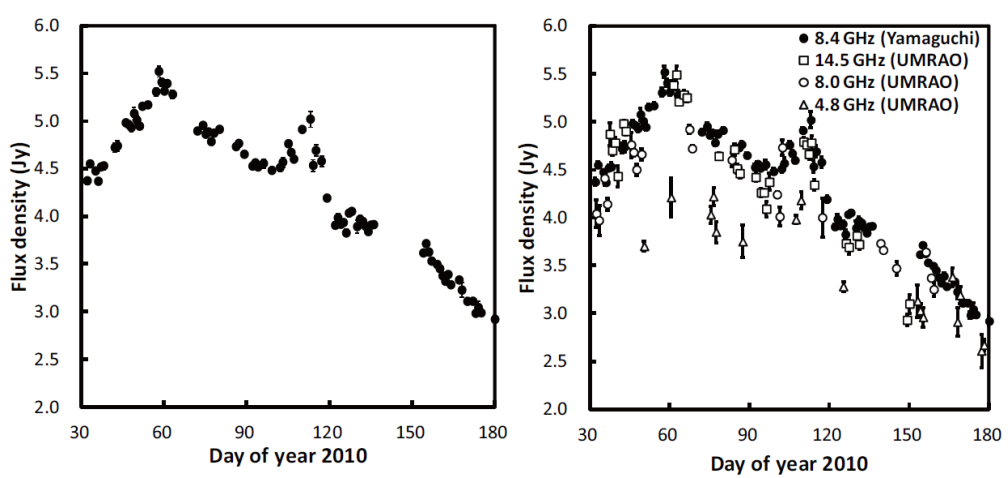

Figure 1: Left: light curve of OT 081 at $8.4 \mathrm{GHz}$ from observations with the Yamaguchi radio telescope. The plotted flux density error represents the standard measurement error for each day. Right: comparison of UMRAO and Yamaguchi light curves. Filled circles: Yamaguchi data at $8.4 \mathrm{GHz}$; open squares: UMRAO data at $14.5 \mathrm{GHz}$; open circles: UMRAO data at $8.0 \mathrm{GHz}$; open triangles: UMRAO data at $4.8 \mathrm{GHz}$.

(DOY 32), the flux density increased, reaching a maximum value of $5.52 \mathrm{Jy}$ on DOY 58. After that, it decreased, with a lower value of $2.92 \mathrm{Jy}$ at the end of the observing period (DOY 180). Although the decrement was almost monotonic, a small rise and decay is detected around DOY 113. This isolated small flare is referred to as short-term flare in the rest of the paper.

We compared our data with those from UMRAO. Figure $\square$ (right) shows the UMRAO light curves of OT 081 at 4.8, 8.0 and 14.5 GHz from DOY 32 to DOY 180, overlapped with the Yamaguchi light curve at $8.4 \mathrm{GHz}$. The flux density at the three frequencies peaks around DOY 60, with variation patterns similar to those observed with Yamaguchi. The short-term flare is also seen in the UMRAO data, at all three frequencies, even though the sampling at 4.8 and $8.0 \mathrm{GHz}$ is sparse.

\subsection{Properties of the short-term flare}

The light curve in Fig. 1 shows a long-term rise and decay of the flux density at all bands with a short-term flare superimposed on this long-term evolution. In order to separate the short-term flare from the long-term evolution and to investigate properties of the flare quantitatively, we followed the analysis of Abdo et al. [四] and used a function with double exponential forms to fit the observed rise and decay of the flux density. This function is expressed by

$$
F(t)=2 F_{0}\left(e^{\left(t_{0}-t\right) / t_{r}}+e^{\left(t-t_{0}\right) / t_{d}}\right)^{-1},
$$

where $F_{0}$ measures the amplitude of the flare, $t_{0}$ describes approximately the time of the peak (it corresponds to the actual maximum only for symmetric flares, see Eq. 3.2 below), and $t_{r}$ and $t_{d}$ are the rise and decay time, respectively. The peak flux density $F_{\max }$ is reached at time $t_{\max }$ given by

$$
t_{\text {max }}=t_{0}+\frac{t_{r} t_{d}}{t_{r}+t_{d}} \ln \left(\frac{t_{d}}{t_{r}}\right),
$$

which is equal to $t_{0}$ for $t_{d}=t_{r}$ (case of symmetric flares). The duration of the flare is defined as the time during which the flux density is greater than $10 \%$ of the peak value.

The fitting was done separately for each light curve. Since the UMRAO data at 4.8 and 8.0 GHz are limited, only the long-term evolution was considered for those two light curves. In 
Table 1: Parameters modeling the light curve of OT 081 on the long term

\begin{tabular}{lccc}
\hline \multicolumn{1}{c}{ Data } & $\begin{array}{c}t_{d} \\
(\text { days })\end{array}$ & $\begin{array}{c}t_{\max } \\
(\mathrm{DOY})\end{array}$ & $\begin{array}{c}F_{\max } \\
(\mathrm{Jy})\end{array}$ \\
\hline Yamaguchi $8.4 \mathrm{GHz}$ & 209 & 58 & 5.2 \\
UMRAO 14.5 GHz & 159 & 56 & 5.3 \\
UMRAO $8.0 \mathrm{GHz}$ & 229 & 61 & 4.8 \\
UMRAO $4.8 \mathrm{GHz}$ & 178 & 75 & 4.0 \\
\hline
\end{tabular}

Note. The rise time $t_{r}$ is not shown because of the large uncertainty in the determination of this parameter.

Table 2: Parameters modeling the short-term flare observed in OT 081

\begin{tabular}{cccc}
\hline \multicolumn{1}{c}{ Data } & $\begin{array}{c}\text { Duration } \\
\text { (days) }\end{array}$ & $\begin{array}{c}t_{\max } \\
(\mathrm{DOY})\end{array}$ & $\begin{array}{c}F_{\max } \\
(\mathrm{Jy})\end{array}$ \\
\hline Yamaguchi $8.4 \mathrm{GHz}$ & $19.3 \pm 3.3$ & $111.7 \pm 1.9$ & $0.73 \pm 0.13$ \\
UMRAO 14.5 GHz & $23.9 \pm 2.7$ & $111.5 \pm 1.1$ & $0.78 \pm 0.16$ \\
\hline
\end{tabular}

practice, this was accomplished by excluding the measurements at the time of the short-term flare (from DOY 90 to DOY 130). Conversely, the Yamaguchi data at $8.4 \mathrm{GHz}$ and those of UMRAO at 14.5 GHz include many points, permitting both the long-term trend and the short-term flare to be modeled simultaneously. The parameters of the fits characterizing the short- and long-term changes are given in Tables $\square$ and $\square$, respectively. Figure $\square$ plots the corresponding fitted functions.

Examination of the results from the fits shows that the flux density on the long-term peaks near DOY 60 with a decay time of about 200 days. Taking an additional step, the short-term flare was isolated by subtracting the long-term model from the observed light curves, as illustrated in Fig. [1]. The plot in this figure shows that the flare lasts about 20 days, in agreement with the durations in Table $\square(19.3 \pm 3.3$ days at $8.4 \mathrm{GHz}$ and $23.9 \pm 2.7$ days at $14.5 \mathrm{GHz})$. The peak times at the two frequencies are consistent (DOY 111.7 at $8.4 \mathrm{GHz}$ and DOY 111.5 at $14.5 \mathrm{GHz}$ ), indicating that variations are synchronized. Peak flux density is $0.73 \mathrm{Jy}$ at $8.4 \mathrm{GHz}$ and is $0.78 \mathrm{Jy}$ at $14.5 \mathrm{GHz}$.

Comparing with the short-term flare observed for PKS 1510-089 [四], the duration of the flare is shorter for OT 081 (20 days vs 30 days for PKS 1510-089). Moreover, the flare for PKS 1510-089 showed a time lag between 8.4 and $22 \mathrm{GHz}$. This indicates that the flare occurred in an optically thick region since the peak time is expected to be delayed at the lower frequencies in such a case. By contrast, the short-term flare for OT 081 must have occurred in an optically thin region since variations between 8.4 and $14.5 \mathrm{GHz}$ were found to be synchronized, as noted above.

Based on the properties of the short-term flare observed in OT 081, we may constrain the intrinsic size of the corresponding emitting region, $R_{\mathrm{stf}}$, using a relation of causality, as given by the following equation:

$$
R_{\mathrm{stf}} \leq c t_{\mathrm{var}} \frac{\delta}{(1+\mathrm{z})}
$$

where $\delta$ is the relativistic Doppler factor, $\mathrm{z}$ is the redshift, and $t_{\mathrm{var}}$ is the time scale of variability. Assuming $\delta=5$ [目] and $t_{\mathrm{var}}=t_{r}$, we derive a value $\leq 0.01 \mathrm{pc}$ for $R_{\mathrm{stf}}$. The size of the region 

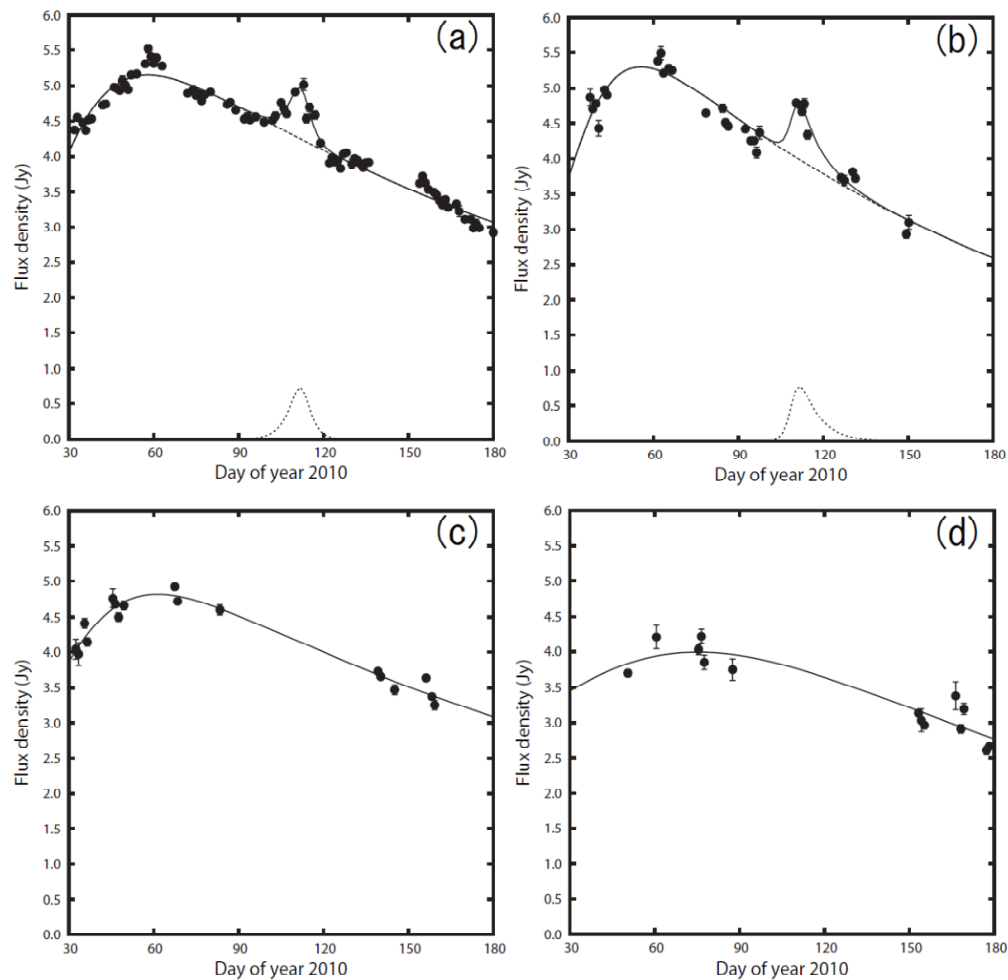

Figure 2: Light curves of OT 081 and fitted functions: a) Yamaguchi data at $8.4 \mathrm{GHz}$; (b) UMRAO data at 14.5 GHz; (c) UMRAO data at 8.0 GHz, (d) UMRAO data at $4.8 \mathrm{GHz}$. The fitted functions are shown as dotted lines (short-term flare), dashed lines (long-term evolution) or solid lines (combined models).

emitting the short-term flare is thus much smaller than the size of VLBI core [目]. This small size means that the brightness temperature in the flaring region should be high, a favorable condition for $\gamma$-ray emission to arise. In this respect, we plan to study further the origin of the short-term flare by using both VLBI and Fermi data and present our results in a future paper (Ishida et al. in prep.).

\section{Summary}

We have conducted daily monitoring of the BL Lac object OT 081 at $8.4 \mathrm{GHz}$ with the Yamaguchi $32 \mathrm{~m}$ radio telescope from February to June 2010. The purpose of this monitoring was to search for intrinsic variability on time scales shorter than a month. Based on these data, we detected a short-time flare with a duration of 19.3 days and an amplitude of $0.73 \mathrm{Jy}$, overlapped on a long-term trend with a time scale of about 200 days. This short-term flare is also seen in the UMRAO data at 4.8, 8.0, and $14.5 \mathrm{GHz}$. We extracted the short-term flare by subtracting the long-term trend from the data, allowing us to study the flare in further details. The flare was found to occur synchronously at 8.4 and $14.5 \mathrm{GHz}$, suggesting that the flaring region was optically thin at 8.4 and 14.5 GHz. The duration of the flare indicates that the emitting region is smaller than $0.01 \mathrm{pc}$. 


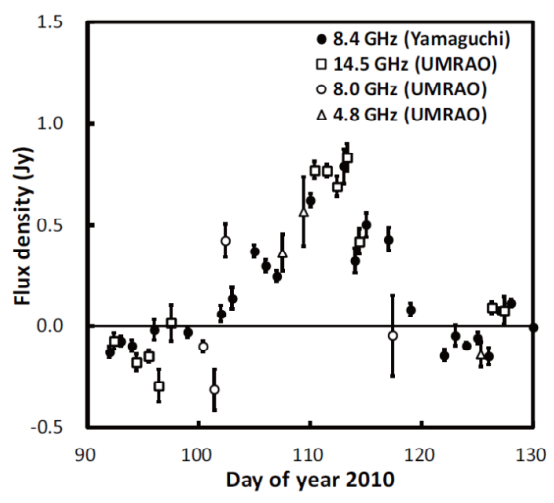

Figure 3: Light curve of OT 081 with focus on the period of the short-term flare. Filled circles: Yamaguchi data at $8.4 \mathrm{GHz}$; open squares: UMRAO data at $14.5 \mathrm{GHz}$; open circles: UMRAO data at 8.0 GHz; open triangles: UMRAO data at $4.8 \mathrm{GHz}$.

\section{Acknowledgments}

This work was partially supported by Grant-in-Aid for Scientific Researches (24540240, M.K.) from the Japan Society for the Promotion of Science. UMRAO is supported in part by a series of grants from the NSF and by funds from the University of Michigan Department of Astronomy. Takafumi Ishida would like to thank the organizers of the conference for providing financial support (through the RadioNet3 programme) to attend the symposium and present this paper.

\section{References}

[1] Abdo, A. A., Ackermann, M., Ajello, M., et al. 2010, ApJ, 722, 520

[2] Aller, M. F., Hughes, P. A., \& Aller, H. D. 2010, arXiv:1007.0258

[3] Dallacasa, D., Stanghellini, C., Centonza, M., \& Fanti, R. 2000, A\&A, 363, 887

[4] Fan, J. H., \& Lin, R. G. 1999, ApJS, 121, 131

[5] Kadota, A., Fujisawa, K., Sawada-Satoh, S., Wajima, K., \& Doi, A. 2012, PASJ, 64, 109

[6] Lu, R.-S., Shen, Z.-Q., Krichbaum, T. P., et al. 2012, arXiv:1204.4150

[7] Marscher, A. P., Jorstad, S. G., Larionov, V. M., et al. 2010, ApJL, 710, L126

[8] Ott, M., Witzel, A., Quirrenbach, A., Krichbaum, T. P., Standke, K. J., Schalinski, C. J., \& Hummel, C. A. 1994, A\&A, 284, 331

[9] Sasada, M., Uemura, M., Fukazawa, Y., et al. 2011, PASJ, 63, 489

[10] Tanihata, C., Urry, C. M., Takahashi, T., et al. 2001, ApJ, 563, 569

[11] Torniainen, I., Tornikoski, M., Teräsranta, H., Aller, M. F., \& Aller, H. D. 2005, A\&A, 435, 839 